\title{
Electroconvulsive therapy machines
}

\section{Carol Robertson \& Grace Fergusson}

Electroconvulsive therapy (ECT) is a long established form of treatment for specific, serious mental disorders, but one which is still surrounded by much misunderstanding and fear. The use of electrical current administered to the brain to 'treat' illness remains a barbaric concept to many people. Much research has been conducted to establish the efficacy of ECT, which is now widely accepted. However, the working principles of the ECT machine itself are little understood, and more research remains to be done to establish, more scientifically, which is the 'best' way to administer the electrical stimulus. With the recent introduction of the concept of "stimulus dosing" (for a review see Lock, 1994), the need to be aware of the ECT machines available and their abilities and limitations is important.

This article is intended as a guide to consultants, on the machines which are currently available and will indicate their strengths and weaknesses.

\section{History of ECT machines}

It is now 57 years since Cerletti and Bini introduced their novel technique of inducing therapeutic seizures by electrical stimulation (Cerletti \& Bini, 1938). The first generation ECT machines utilised sine-wave electricity and were of increasingly simple design, resulting in virtually a 'black box', connected to the mains with a transformer and two wires inside leading to two electrodes. The energy delivered could be altered by varying either a voltage or current switch, and the treatment time by manual depression of the 'treat' button and counting. Early treatment was always given bilaterally and unmodified by anaesthetic and it soon became apparent that as well as beneficial effects, ECT resulted in temporary post treatment confusion and memory loss.

By the mid 1940s it had been shown that seizures could be produced more efficiently using an interrupted electrical stimulus and that sideeffects, such as disorientation, were less severe. This observation was overshadowed by the introduction of general anaesthesia for ECT in the 1950 s and it was not until the 1970s that there was a renewal of interest in the production of ECT machines (Weaver et al, 1974). The new machines delivered pulsed electrical waveforms and from the 1980 s contained a capacitor to allow for delivery of constant charge as opposed to constant voltage (refer to the section on physical facts for more detail). By this time there had been much debate as to the preferred method of electrode placement either bilateral or unilateral (Weiner, 1980).

The eventual arrival of machines which delivered brief pulse instead of sine-wave energy encouraged researchers to minimise the amount of electricity which would induce a seizure. This was done in the belief that any observed fit was both necessary and sufficient. Coupled with the method of using unilateral ECT this became accepted as the best practice, until clinicians became aware that this form of ECT was no longer such an effective treatment (Weiner, 1980). There followed a decline in the use of ECT which may have been as much a function of the underpowered machines available as the technique used. In 1982, Robin \& De Tissera concluded that the minimum amount of electricity needed for therapeutic effect was somewhere above the seizure threshold for the individual. The debate regarding this aspect of treatment continues (Lock, 1994).

Recent research has highlighted the need to determine what is the therapeutic ingredient of the ECT stimulus. Several machine manufacturers have now introduced independent variation of stimulus parameters and integrated EEG monitors. The traditional quantification of dose administered in terms of milliCoulombs (mC), may no longer be sufficient, as other stimulus parameters may alter efficacy and side-effects of treatment. Thus some of the modern machines allow for adjustment of the current, pulse width, pulse frequency and

Carol Robertson is a Senior Registrar at Royal Cornhill Hospital, Cornhill Road, Aberdeen, AB9 2ZH. Grace Fergusson is a Staff grade Psychiatrist at Argyll and Bute Hospital, Lochgilphead, Argyll PA31 8LD. Both are members of the CRAG/SCOTMEG Good Practice Group on ECT, and are affiliated to the Royal College of Psychiatrists' Special Committee on ECT. 
stimulus duration. It should now be possible to measure clinically, and on EEG, the effects of altering just one parameter.

Until recently, the only machines available in the UK were those manufactured by Ectron, Theratronics and Neurotronic Therapy System. However only Ectron now remains in its original form, with Theratronics having ceased trading and Neurotronics changing distributors. Recently American machines, complying with acceptable safety standards, have become available in this country.

\section{Present usage}

In the 1980 review of ECT in Great Britain, which proved to be a milestone for the service, Pippard \& Ellam (1981) established that $28 \%$ of the clinics they visited used obsolete machines. They also indicated that $40 \%$ of clinics did not have their machines regularly maintained. Following the controversy surrounding their publication and the subsequent publication of the Royal College of Psychiatrists' Guidelines (1989), repeat audit revealed that several centres were still employing equipment which was no longer recommended (Pippard, 1992).

A recent survey of ECT services in Scotland (Robertson et al, 1995) identified that a third of the clinics used ECT machines considered obsolete. At the time of the survey, in $1994,42 \%$ of clinics were using the Ectonus 5 . However, several centres were hoping to update their machines to a MECTA machine or the Ectonus 5A. The results of these audits highlights the problems that clinics face in keeping abreast of technological and research developments.

\section{Physical facts}

To deliver appropriate ECT to an individual patient the seizure threshold for that individual must be exceeded (Lock, 1994). However the actual value of the amount of electricity required to do this depends upon the parameters of the stimulus generated, which varies between machines. Knowledge of the basic physics of the electrical stimulation may assist clinicians to select an appropriate device.

\section{Constant current}

Constant current machines maintain a predetermined current level by altering output voltage in line with resistance (impedance). These machines are more accurate and also safer than the older constant voltage machines in which there was a risk of overdose of current in patients with a low skull resistance.

\section{Impedance}

For the purpose of ECT the impedance can be taken as the summation of the resistance conferred by static and dynamic elements. Static impedance is determined by the quality of electrode contact with the patients' skin. Dynamic impedance is a function of the summed electrical properties of the skin, hair, skull, blood vessels, meninges, brain and cerebrospinal fluid. Some machines are calibrated according to an estimated average impedance (usually around $220 \mathrm{Ohms}$ ) but other machines can measure the individual static impedance prior to treatment and alter the output accordingly.

\section{Waveform}

For further descriptions of waveform see Lock (1994). Older machines utilised a sine-wave form of current which resulted in more cognitive sideeffects and was no more efficacious than the modern brief-pulse current (Weiner et al, 1986; Scott et al, 1992). Crescendo or 'soft start' pulses are reputed to modify the initial phase of the convulsion.

\section{Mode}

Unimodal (or uniphasic) stimuli are pulses in only one direction (positive), while bimodal (or biphasic) stimuli are in both positive and negative directions.

\section{Current}

The amplitude of the waveform indicates the maximum current (milliamps; $\mathrm{mA}$ ). A fast changing current results in cells firing at lower intensities of charge. Within a given total charge, increments in current have been shown to be an efficient way of inducing a seizure (Koester, 1985).

\section{Pulse width}

The duration of each pulse of current within the stimulation is the pulse width (milliseconds). Early work suggested that ultra-brief pulses are ineffective (Cronholm \& Ottosson, 1960), while pulses of more than 2 milliseconds may also be inefficient and add to side-effects (Sackeim et al, 1994).

\section{Frequency}

This is a measure of the number of pulses of electricity per second (Hertz, Hz). For bimodal stimuli it refers to the number of pulse pairs per second. Abrams (1994) suggested that the frequency 
Box 1 Minimum requirements for an ECT machine

Constant current output

Delivery of brief pulse energy

Facility for wide output range

Compliance with safety standards.

Additional facilities available include variable output parameters, and EEG monitoring.

should be less than $83 \mathrm{~Hz}$ to prevent unnecessary stimulation of neurones during the refractory period. However if increased penetration to deeper tissue is required for efficacy then it may be that higher frequencies are needed to ensure the phenomenon of temporal summation occurs (Sackeim, 1994).

Time

The time is the total length of stimulation or 'pulse train' (seconds). Weaver et al (1982) considered a time of between 3-5 seconds to be optimal. Ten years later Abrams advocated the use of a 4-8 second stimulus to optimise the effects of unilateral ECT (Abrams, 1992). Other advocates of lengthy stimuli quote the work of Scandinavian investigators who use long stimulus trains without excess cognitive dysfunction (Fromholt et al, 1973). Sackeim et al (1994) agree that increasing the time may be an efficient way of increasing the stimulus dose, but caution against having the facility for long stimulations on ECT machines. Sackeim et al (1991) observe the onset of seizure activity as early as one second into treatment (especially with high total charge) and warns that continuing to stimulate during depolarisation is both inefficient and potentially detrimental.

\section{Charge $v$. energy}

Attempts to quantify the total stimulus or 'dose' in ECT rely on Ohms law:

$$
\text { Voltage }=\text { current } \mathrm{x} \text { resistance. }
$$

With ECT it is essential to integrate the parameters over time in order to provide a summary unit. There has been a move away from using the term 'energy' (Maxwell, 1968) to the unit of charge, which refers to the quantity of electrons transferred over a given period (Sackeim et al, 1987; Weiner $e t$ al, 1987). The unit of charge is the coulomb (C) or millicoulomb $(\mathrm{mC})$ and the total is calculated by multiplying all electrical parameters together which gives a measure of amps $x$ time to signify 'dose':
Current (A) $x$ pulse width (milliseconds) $x$ frequency $(\mathrm{Hz}) \times$ time (seconds) $\times 2$ (if bimodal).

In summary therefore, it is suggested that the physical properties of the ECT stimulus affect both efficiency and side-effects. Clarification of the precise way in which these parameters exert their effect is needed in order to direct machine manufacturers towards the production of the 'smart' ECT machine, which does not yet exist.

\section{Choosing a machine}

The resurgence of interest in ECT has resulted in the manufacture of more sophisticated equipment. Thus the choice of machine available to a clinician considering new equipment is wider than was previously the case. The 'right' machine for a centre must be judged on the requirements and resources of the ECT clinic. The onus is on the clinician to ensure that their ECT clinic has up-to-date equipment.

All machines now available in the UK are of the constant current brief pulse variety. However, several questions should be considered prior to purchasing a new machine.

Is there an adequate range of output to allow for the treatment of patients whose seizure threshold lies at the extremes?

(i) Young females may have a seizure threshold below $50 \mathrm{mC}$.

(ii) Elderly men at the end of their course of treatment may require a dose in excess of $700 \mathrm{mC}$ to exceed the seizure threshold which rises with ECT.

(iii) It has been suggested that there may be as much as a 40-fold inter-individual variation in seizure threshold (Sackeim et al, 1987).

Do I want a simple 'single dial' unit or would I prefer to have the facility to allow for variation of individual machine parameters?

(i) Flexibility puts the onus on the psychiatrist to determine treatment variables. Simplicity is restrictive but increases ease of use (Weaver \& Williams, 1987).

(ii) The current knowledge of 'ideal' stimulus parameters may not yet allow for an accurate single dial unit.

Does the machine test the skull impedance?

(i) Direct measurement of skull impedance allows this to be included in proportional adjustments to voltage in order to maintain a constant current. Machines without this facility vary voltage according to an estimated patient impedance. 
(ii) However, the resistance of the head will vary depending on the electrical parameters used and the resistance of the circuit is determined by the adequacy of contact and factors other than the skull impedance.

Would EEG monitoring improve the practice of ECT?

As yet there is not enough evidence to be definitive about the routine use of EEG monitoring in ECT. In favour

(i) More accurate measurement of seizure duration

(ii) Possible detection of missed status epilepticus (Scott \& Riddle, 1989)

(iii) Post-treatment EEG suppression may be a Against marker for adequacy (Krystal et al, 1993).

(i) The Hamilton cuff method of measuring seizure length correlates highly with EEG measures and is easier and less expensive (Fink \& Johnson, 1982)

(ii) Poor interrater reliability of EEG seizure duration (Ries, 1985)

(iii) Inaccuracy of two-channel EEG with respect to generalised ictal activity (Warmflash et al, 1987)

(iv) EEG changes of post ictal suppression may not be specific or sensitive enough to be clinically useful in determining efficacy (Sackeim et al, 1994);

(v) Doctors concerned with reading the EEG have to be trained and competent (Scott et al, 1989)

(vi) EEG recording of seizure activity is no substitute for good ECT practice (Scott $e t$ al, 1989).

Given the above, the additional expense of buying the EEG monitor and the running costs, it is suggested that at present EEG monitoring is reserved for research purposes (Royal College of Psychiatrists, 1995).

Is the dose delivered by the machine, displayed?

(i) If not, then dose administered may need to be calculated manually, and depending on impedance, this may not be the actual dose delivered.

Does the machine comply with British Safety Standards or equivalent?

(i) Additional safety standards are available on some machines, such as audible and visual indication of stimulus delivery.

(ii) An automatic stimulus abort feature is available on some machines such that the treatment only continues when the 'treat' button is depressed. Other machines continue delivery to the end of the stimulus once the button is initially depressed. (iii) The position of the treat button may be on the electrodes and the machine or only on the machine. If the treat button is only on the machine then a third party is required to depress the button on the instruction of the treating psychiatrist.

Does the company offer back-up and after-sales service?

(i) Are there local facilities for regular maintenance or is a service contract required?

How much does the machine cost?

(i) Matters of cost cannot be ignored and should be considered in the light of budget and priorities within the unit. However the relative cost of an ECT machine is minimal when compared to anaesthetic or resuscitation equipment. The costs of servicing and replacing machines must be included in the running costs of an ECT suite.

\section{Manufacturers and machines}

Each of the machines suitable and available in the UK will be described in turn (giving technical details and cost, at June 1995, for reference). The manufacturers/suppliers' addresses will be given in the reference section.

All the machines feature hand held electrodes and all have British Standard 5724 or EU equivalent.

\section{Ectron}

Ectron Limited has been the principal British manufacturer of ECT machines for many years and has produced a series of models over this time. The most popular machine in the UK was the Series 5, which produced a constant current brief pulse stimulus. However due to the limited range of stimuli available with this model $(150-400 \mathrm{mC})$ and fixed stimulus duration ( 3.25 seconds), an updated model was introduced in 1993. The Series 5A Ectonus has a wider range of stimuli available and a variable stimulus duration. The company also produce a Series $5 \mathrm{~A}$ Ectonustim which, although identical to the Ectonus, has the additional feature of an optional "non-convulsive cerebral stimulation". This low voltage unidirectional sine-wave stimulus is suggested for use "to reduce amnesia or confusion after ECT" or "to give a painful stimulus in conjunction with therapeutic suggestions in the treatment of hysteria". The authors are unaware of the usefulness of this function. 


\section{Table 1. Comparison of the ECT machines currently in use in the UK}

$\begin{array}{lllll}\text { Machine } & \begin{array}{l}\text { Output } \\ \text { range }(\mathrm{mC})\end{array} & \begin{array}{l}\text { Output } \\ \text { control }\end{array} & \begin{array}{l}\text { Output } \\ \text { display }\end{array} & \begin{array}{l}\text { EEG } \\ \text { monitoring }\end{array} \\ \text { ECTONUS 5A } & 50-700 & \text { Single dial } & \text { Yes } & \text { No } \\ \text { NTS-R } & 75-4455 & \text { Multiple dial } & \text { No } & \text { No } \\ \text { NTS-C } & 60-720 & \text { Single dial } & \text { No } & \text { No } \\ \text { MECTA JR1 } & 22-1152 & \text { Multiple dial } & \text { Yes } & \text { No } \\ \text { MECTA SR1 } & 22-1152 & \text { Multiple dial } & \text { Yes } & \text { Yes } \\ \text { MECTA JR2 } & 25-1200 & \text { Single dial } & \text { Yes } & \text { No } \\ \text { MECTA SR2 } & 25-1200 & \text { Single dial } & \text { Yes } & \text { Yes } \\ \text { THYMATRON DGx } & 25-1008 & \text { Single/multiple dial } & \text { Yes } & \text { Optional }\end{array}$

\section{Ectonus 5A}

Output control - Single dial (with inner scale for small dose alterations)

Price - $£ 2900$ (Ectonustim $£ 3100$ ) plus VAT

Waveform - Split uniphasic brief pulse

Pulse frequency - Automatically varies; $30-70 \mathrm{~Hz}$ (in groups of three pulses giving $90-210 \mathrm{~Hz}$ pulses)

Pulse width - Fixed combined 2.2 milliseconds

Output range $-50-700 \mathrm{mC}$

Current $-750 \mathrm{~mA}$

Stimulus duration - Automatically varied $1.25-6.25$ seconds

Trigger operation - Machine or electrode

Output display -Actual dose delivered displayed on digital panel

Monitoring - None

Safety features - Stimulus passage indicated by visual and audible signal. No automatic stimulus abort feature

Special features - Has test function for static impedance. Has 'Auto-Crescendo' function for "gentle onset of treatment"

Servicing - Easily available

Remarks - This model provides a larger range of stimuli than previous machines, but may not provide a complete range for patients with very high seizure thresholds. Little use for small inner output dial. Usefulness of 'Auto-Crescendo' with modern modified ECT doubtful. No scope for independent control of stimulus parameters or additional monitoring. Pulse width and frequency may be too long. Style of machine is familiar to many.

Ectonus 5B available from late 1995. Identical to $5 \mathrm{~A}$ but has additional display of patients' dynamic impedance.

\section{SLE}

The Medical Physics Department of the Northern Regional Health Authority developed the Neurotronic machine. Recently SLE took over the marketing of these machines and now produce two models - the NTS-R and the NTS-C. The NTS-R is based on the previous research model and allows for independent alteration of current, frequency, stimulation time, pulse width and phasic mode of stimulation. The NTS-C is a simpler machine allowing for control of only the current administered.

NTS-R

Output control - Five dial control

Price - $€ 3450$ plus VAT

Waveform - Brief pulse; uniphasic or biphasic

Pulse frequency - Three settings; 50,100 or $150 \mathrm{~Hz}$

Pulse width - Three settings; $1.5,2$ or 2.5 milliseconds

Output range $-7.5-4455 \mathrm{mC}$

Current $-100-990 \mathrm{~mA}$ in $10 \mathrm{~mA}$ steps

Stimulus duration - Six settings; $1-6$ seconds

Trigger operation - Machine or electrode

Output display - None

Monitoring - None

Safety features - Only has audible signal during current passage. No automatic stimulus abort function

Special features - No test function for static impedance, but has warble tone if unable to maintain current. Has 'soft start' feature to raise the current progressively over 1 second Servicing - Unsure

Remarks - Little guidance is available regarding a suitable dosing strategy for this machine and operators have to calculate the stimulus administered in $\mathrm{mC}$ from all the variables which can be altered. No substantial evidence that the 'soft start' is necessary. Maximum stimulus available $(4455 \mathrm{mC})$ is very high and great care would be needed to ensure this was not inappropriately administered. The machine is complicated to use but may provide adequate parameter adjustment for research purposes, particularly as it allows for adjustment of current. Voltage setting may be too low to allow use of higher output settings.

\footnotetext{
NTS-C

Output control - Single dial control

Price - $£ 3250$ plus VAT

Waveform - Brief pulse; biphasic only

Pulse frequency - Fixed $100 \mathrm{~Hz}$

Pulse width - Fixed 1 millisecond

Output range $-60-720 \mathrm{mC}$ (dial quotes $\mathrm{mA}$ )

Current $-100-1200 \mathrm{~mA}$ in $10 \mathrm{~mA}$ steps

Stimulus duration -3 seconds fixed
} 
Trigger operation - Machine or electrode

Output display - None

Monitoring - None

Safety features - Only has audible signal during current passage. No automatic stimulus abort function

Special features - No test function for static impedance, but has warble tone if unable to maintain current. Has 'soft start' feature to raise the current progressively over one second

Servicing - Unsure

Remarks - Little guidance is available regarding a suitable dosing strategy for this machine, output tables not provided (output dial set in $\mathrm{mA}$ ). No substantial evidence that the 'soft start' is necessary. Slightly limited output range for patients with a very high seizure threshold. The fixed pulse frequency of $100 \mathrm{~Hz}$ may be excessive.

\section{MECTA}

The ECT machines manufactured by the MECTA Corporation are the most popular in North America. Since 1992 these machines have been marketed in the UK by Medelec Vickers Limited. The machines currently available are the JR1, SR1, JR2 and SR2. The units are built on a 'modular' basis, allowing a degree of flexibility. The basic JR range has no monitoring functions, whereas the $S R$ range has a built-in dual channel monitor. The $S R$ machines allow for single channel ECG or EEG monitoring or for dual channel EEG monitoring. The JR range can be upgraded by the addition of the monitoring module. The series 1 models have independent control of brief pulse stimulus parameters for current, pulse width, frequency and stimulus duration, while the Series 2 models have a predetermined single output control dial. USA domestic and international machines are available, but discussion will be confined to the British models now marketed.

\section{JR1/SR1}

Output control - Four dial control

Price - JR1 is $£ 4052$ plus VAT SR1 is $€ 7923$ plus VAT

Waveform - Biphasic brief pulse

Pulse frequency - Six settings; $40-90 \mathrm{~Hz}$

Pulse width - Six settings; 1-2 milliseconds

Output range $-22-1152 \mathrm{mC}$

Current - Six settings; $550-800 \mathrm{~mA}$

Stimulus duration - Ten settings $0.5-4.0$ seconds

Trigger operation - Machine or electrode

Output display - Outcome of self-test, functional status, set dose and dose administered are displayed. SR model prints information

Monitoring - EEG/ECG on SR models

Safety features - Visual and audible signal prior to and during current passage. Automatic stimulus abort function Special features - Has test function for static impedance. Electrodes use paste or gel not fluid
Servicing - Unsure, but it is a large medical instrument company with a servicing network

Remarks - Modular system allows for easier upgrading and modification by changing the microprocessor unit. SR1 is only recommended if there is a specific requirement for and expertise with EEG monitoring. Suitable for centres with specific research interests in brief pulse stimulation characteristics. Complicated array of stimulus controls. Dose titration guides available. Large range of stimulus available.

JR2/SR2 models

Output control - Single dial

Price - JR2 is $€ 4052$ plus VAT

SR2 is $€ 7923$ plus VAT

Waveform - Biphasic brief pulse

Pulse frequency - Automatically varies; $40-90 \mathrm{~Hz}$

Pulse width - Automatically varies; $1-1.4$ milliseconds

Output range $-25-1200 \mathrm{mC}$ ( 25 and $50 \mathrm{mC}$ increments)

Current $-550-800 \mathrm{~mA}$ (automatically varied)

Stimulus duration - 0.55-6.00 seconds (automatically varied)

All other features are as JR1/SR1 models above.

Remarks - Modular system allows for easier upgrading and modification by changing the microprocessor unit. SR2 only recommended if there is a specific requirement for and expertise with EEG monitoring. No scope for independent control of stimulus parameters. Usefulness of ECG monitor doubtful. Simple one dial operation. Large range of stimulus available.

\section{Somatics}

The Thymatron machine was developed in the USA and is the second most popular machine in North America. The machine has recently been marketed in the UK by Dantec Limited. The Thymatron is similar to the MECTA machines in having two modules - a treatment unit and a monitor unit; the microprocessor can also be upgraded without changing the entire machine. The machine is supplied with a 'flexidial' which can be fitted to allow for adjustment of pulse width or frequency (by increasing stimulus duration) while charge remains fixed. This dial also allows for application of the ' $x 2$ Energy' function which doubles the output range to $50-1008 \mathrm{mC}$ and requires care to remove alarm and recalculate dose administered.

Thymatron DGx

Output control - Single dial control percentage of total output (options on flexidial mode and $\times 2$ Energy)

Price - $€ 8450$ plus VAT - with EEG printer E5550 plus VAT - without EEG printer

Waveform - Biphasic brief pulse

Pulse frequency - Automatically varied $30-70 \mathrm{~Hz}$ (variable $30-70 \mathrm{~Hz}$ in flexidial mode, fixed $70 \mathrm{~Hz}$ on $\times 2$ Energy) 
Pulse width - Fixed 1 milliseconds (0.5-1.5 milliseconds Flexidial mode, fixed 1.5 milliseconds on $x 2$ Energy) Output range $-25-504 \mathrm{mC}(50-1008 \mathrm{mC}$ on $\times 2$ Energy) Current - Constant $900 \mathrm{~mA}$

Stimulus duration $-0.47-4$ seconds (up to 8 seconds in Flexidial mode)

Trigger operation - On machine only

Output display - Actual dose delivered is displayed Monitoring - Facility for EEG/EMG/ECG monitoring. EEG has audible signal. Monitor prints EEG/EMG end point

Safety features - Has audible and visual signal during current passage and a pre-treatment warning. Two automatic stimulus abort functions - one on treatment button the other automatic if current administered is going to vary by more than $5 \%$ from selected value. Alarm for extended seizures Special features - Has test function for static impedance. 'Post-ictal Suppression Index' is a suggested method of predicting clinical improvement - by computer reading of EEG activity

Servicing - By Dantec Electronics UK. Unsure

Remarks - Claims of "Just set to your patients age and treat" are calculated at dose 2.5 times average seizure threshold for patients age and were calculated for patients receiving unilateral ECT. Dosing strategy for bilateral ECT now produced. EEG monitoring and 'Post-ictal Suppression Index' not a substitute for clinical judgement - requires further evaluation. The flexidial allows for more flexibility if required. Energy $x 2$ option allows wide range of output but must be used with care. Available with or without EEG printer monitor.

\section{Obsolete machines}

With changing technology and progressive research certain previously available ECT machines are no longer acceptable. The probable maximum shelf life of a machine may be around five years. Most comparable anaesthetic and resuscitation equipment is regularly renewed.

\section{Ectron}

All Series 2 machines - Underpowered

All Series 3 machines - Underpowered (even after modification)

All Series 4 machines -Sine-wave model; unacceptable side effects

Duopulse model - Underpowered

(Series 5 Ectonus - Output range may be too small for dose titration if this technique is used).

\section{Siemens}

Konvulsator-Chopped sine-wave - unacceptable side effects failed safety standard (BS 5724)

Theratronics

Transpsychon - failed safety standard (BS 5724)

\section{Royal College of Psychiatrists' recommendations}

The Royal College of Psychiatrists' Guidelines on the administration of ECT, 1995, includes a chapter on ECT machines. This is a comprehensive guide to the machines available and has ranked seven criteria for each machine on various aspects of suitability. The College has not recommended one single machine, but has shortlisted the following as being "suitable for routine clinical practice": MECTA SR2; MECTA JR2; Thymatron DGx; Ectonus 5A

\section{Conclusions}

The process of administering ECT is now becoming more technical and the ECT machines developed reflect these changes. There is, however, no single ideal machine and those discussed in this article all have advantages and disadvantages which may change over time as research progresses. Interdisciplinary collaboration between psychiatrists practising ECT and electrical engineers is needed to determine the precise stimulus parameters for effective delivery of ECT (Gangadhar et al, 1994).

One major factor in the selection of an appropriate machine is the requirements of the ECT service, since the most flexible and complicated machine may not be the most appropriate. The education of junior staff administering treatment, with regular updating and clarification, is essential to any clinic, as is the continued interest and support of consultant staff. An understanding of the underlying principles of the machine can only help to improve the quality of ECT services.

\section{Acknowledgements}

The authors are grateful to Dr Toni Lock for information regarding ECT machines. Dr Chris Freeman has supported the authors in their continued interest in ECT. Professor I. Reid and Dr A. V. P. MacKay have assisted in the preparation of this manuscript.

\section{Manufacturers/suppliers}

Dantec Electronics Ltd, Garonor Way, Royal Portbury, Bristol BS20 9XE. Telephone: 01275375333

Ectron Ltd, Knap Close, Letchworth, HertsSG6 1AQ. Telephone: 01462682124

SLE Ltd, Twin Bridges Business Park, 232 Selsdon Road, South Croydon, Surrey CR2 6PL. Telephone: 01816811414

Medelec Vickers Ltd, Manor Way, Old Woking, Surrey GU22 9JU. Telephone: 01483770331 


\section{References}

Abrams, R. (1992) Electroconvulsive Therapy (2nd Edn). Oxford: Oxford University Press.

- (1994) Commentary: stimulus parameters and efficacy of ECT. Convulsive Therapy, 10, 124-128.

Cerletti, V. \& Bini, L. (1938) Un nuevo metodo di shockterepie; L'elettro-shock. Boll Acad Med Roma, 64, 136-138.

Cronholm, B. \& Ottosson, J. O. (1960) Experimental studies of the therapeutic action of electroconvulsive therapy in endogenous depression. Acta Psychiatrica Scandinavica, 35, 69102.

Fink, M. \& Johnson, L. (1982) Monitoring the duration of electroconvulsive therapy seizures. Archives of General Psychiatry, 39, 1189-1191.

Fromholt, P., Christensen, A. L. \& Stromgren, L. S. (1973) Effects of unilateral and bilateral electroconvulsive therapy on memory. Acta Psychiatrica Scandinavica, 46, 466-478.

Gangadhar, B. N., Janakiramaiah, N. \& Candade, V. S. (1994) Towards another generation of ECT devices? Convulsive Therapy, 10, 129-130.

Koester, J. (1985) Voltage gated channels and the generation of the action potential. In Principles of Neural Science (eds E. R. Kande \& J. H. Schwartz), pp. 75-86. New York: Elsevier.

Krystal, A. D., Weiner, R. D., McCall, W. V., et al (1993) The effects of ECT stimulus dose and electrode placement on the ictal electroencephalogram: An intra-individual crossover study. Biological Psychiatry, 34, 759-769.

Maxwell, R. D. (1968) Electrical factors in electroconvulsive therapy. Acta Psychiatrica Scandinavica, 44, 436-448.

Lock, T. (1994) Advances in the practice of electroconvulsive therapy. Advances in Psychiatric Treatment, 1, 47-56.

Pippard, J. (1992) Audit of electroconvulsive treatment in two National Health Service regions. British Journal of Psychiatry, $160,621-637$.

- \& Ellam, L. (1981) Electroconvulsive treatment in Great Britain. British Journal of Psychiatry, 139, 563-568.

Ries, R. K. (1985) Poor interrater reliability of Mecta EEG seizure duration measurement during ECT. Biological Psychiatry, 20, 94-119.

Robertson, C., Freeman, C. P. \& Fergusson G. M. (1995) Electroconvulsive therapy services in Scotland. (in press)

Robin, A. \& De Tissera, S. (1982) A double blind controlled comparison of therapeutic effects of low and high energy electroconvulsive therapies. British Journal of Psychiatry, 141, 357-366.

Royal College of Psychiatrists' ECT Sub-Committee of The Research Committee. (1989) The Practical Administration of Electroconvulsive Therapy (ECT). London: Gaskell.

Royal College of Psychiatrists' Special Committee on ECT (1995) The ECT Handbook. CR 39. London: Royal College of Psychiatrists.

Sackeim, H. A. (1994) Response to commentaries: physical properties of the ECT stimulus. Convulsive Therapy, 10, 140152.

-, Decina, P., Portnoy, S., et al (1987) Studies of dosage, seizure threshold and seizure duration. Biological Psychiatry, 22, 249268.

- Devanand, D. P. \& Prudic, J. (1991) Stimulus intensity, seizure threshold and seizure duration: impact on the efficacy and safety of electroconvulsive therapy. Psychiatric Clinics of North America, 14, 803-843.

- Long, J., Liber, B., et al (1994) Physical properties and quantification of the ECT stimulus: 1. Basic principles. Convulsive Therapy, 10, 93-123.

Scott, A. I. F. \& Riddle, W. (1989) Status epilepticus after electroconvulsive therapy. British Journal of Psychiatry, 155, 119-121.

-, Shering, A. \& Dykes, S. (1989) Would monitoring by electroencephalogram improve the practice of electroconvulsive therapy? British Journal of Psychiatry, 154, 853-857.
-, Rodger, C. R., Stocks, R. H., et al (1992) Is old-fashioned electroconvulsive therapy more efficacious? British Journal of Psychiatry, 160, 360-364.

Warmflash, V. L., Sticks, L., Sackeim, H., et al (1987) Reliability and validity of measures of seizure duration. Convulsive Therapy, 3, 18-25.

Weaver, L. A., Ravaris, C., Rush, S., et al (1974) Stimulus parameters in electroconvulsive shock. Journal of Psychiatric Research, 10, 271.

_- Ives, J. \& Williams, R. (1982) Studies in brief pulse electroconvulsive therapy: the voltage threshold interpulse interval, and pulse polarity parameters. Biological Psychiatry, 17, 1131-1143.

-_- \& Williams, R. N. (1987) ECT: Second generation instruments. Biological Psychiatry, 22, 1181-1182.

Weiner, R. D. (1980) ECT and seizure threshold: Effects of stimulus waveform and electrode placement. Biological Psychiatry, 15, 225-241.

-, Rogers, H. J., Davidson, J. R. T., et al (1986) Effects of stimulus parameters on cognitive side-effects. Annals of the New York Academy of Science, 462, 315-325.

-, Weaver, L.A. \& Sackeim, H.A. (1987) Reporting of technical parameters in ECT publication: recommendations for authors. Convulsive Therapy, 4, 88-91.

\section{Multiple choice questions}

1 The optimal ECT machine should:

a be constant current

b be constant voltage

c be brief pulse

d deliver chopped sine-wave energy

e be capable of a wide range of output.

2 The following machines are now considered obsolete:
a Thymatron DGx
b Ectron Duopulse
c Siemens Konvulsator
d Ectron Series 3 - modified
e Theratronics Transpsychon

3 Options to consider before purchasing a new ECT machine include:

a one dial $v$. variable parameters

b colour of casing

c cost of unit

d EEG monitoring

e servicing available.

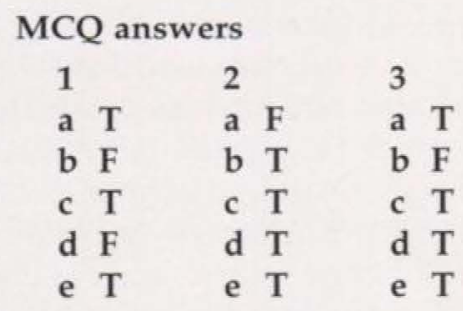

